\section{Basrah Journal Of Surgery}

Bas J Surg, June, 25, 2019

\title{
TRANSLIMBAL INTRAOCULAR ENDOILLUMINATION DURING CATARACT SURGERY
}

\section{Fareed Warid ${ }^{*}$ and Maha Elshafei ${ }^{@}$}

*MB,ChB, CABO, FRCS-Ed, Consultant Ophthalmologist, Department of Ophthalmology, College of Medicine, Basrah University, Basrah, IRAQ. ${ }^{\circledR}$ MD, FRCSI, Senior Consultant Ophthalmologist, Department of Ophthalmology, Hamad Medical Corporation, Doha, Qatar.

\begin{abstract}
The aim of this study is to describe a technique for intraoperative examination of macula and posterior capsule during phacoemulsification surgery in eyes with dense cataract.

This work was done in Ophthalmology Department, Hamad Medical Corporation, Doha-Qatar on 36 patients (42 Eyes) with dense cataract and obscured fundus view. No data were recorded for macular status, and preoperative macular assessment was not conclusive by Ophthalmoscopy, B-Scan Ultrasound, and Optical Coherence Tomography (OCT). All patients were consented for cataract surgery plus additional vitreoretinal surgical procedure if indicated. Fundus examination done during phacoemulsification by translimbal insertion of endo-light probe after Irrigation/Aspiration step prior to lens implantation, capsular-bag expanded by viscoelastic to accommodate probe insert. Posterior capsule status checked by Endolight using microscope lens system only, macula checked by endolight with a vitreoretinal viewing lens system.

Forty-two eyes (25 right and 17 left), (27 males, 15 females) in 39 patients were studied. The mean age was 59 years (47-78 years). Fourteen patients (18 eyes) were diabetics. Preoperative Visual Acuity of $\leq 6 / 60$ was recorded in all patients. Cataract Density was graded by fundus visualization and in all cases, only shadow of optic disc and/or major vessels could be seen. Concurrent intravitreal injections was done in eight eyes (19\%): seven of them were having Diabetic Macular Edema, and one has hemorrhagic Choroidal Neovascular Membrane (CNVM) due to age related macular degeneration. No complication was recorded in relation to Endoillumination. Posterior Capsule visualization was improved significantly and intracapsular lens implantation was done in all cases

In conclusion, translimbal endo-illumination technique improved view to both Macula and posterior capsule during phacoemulsification with subsequent early surgical decision according to endolight findings. No extra incision required.
\end{abstract}

Key words: Surgery, Cataract, Translimbal, Endo-illumination, Phacoemulsification.

\section{Introduction}

Cataract extraction with lens performed surgical procedure worldwide $^{1}$. Up to $29 \%$ of eyes, undergoing cataract surgery may have a co-pathology that can cause a guarded prognosis ${ }^{2}$.

Retinal Pathologies like diabetic retinopathy, age-related macular degeneration, and retinal vein occlusions are aggravated by cataract surgery. Therefore, retinal diseases with a potential for progression should be evaluated and treated prior to cataract surgery $^{3,4}$. Careful preoperative planning, attention to details during surgery, and close postoperative follow-up are essential, as these eyes may have limited visual improvement following cataract surgery ${ }^{5,6}$. 
In dense cataract, preoperative evaluation of the retina could be incomplete, unrecognized retinal pathology may be a cause of unsatisfactory visual outcome following cataract operation and certain retinal diseases may exacerbated by cataract surgery. Multiple retinal conditions may affect cataract surgery outcome, including disorders such as: Diabetic macular edema (DME), Retinal vein occlusion (RVO), Uveitis, Epiretinal membrane formation and peripheral retinal lesions. Peripheral retinal lesions that may be relevant to postoperative outcome include lattice degeneration, retinal breaks, operculated holes, cystic retinal tufts and the whole family of pathologies related to vitreous traction ${ }^{7,8}$.

Posterior segment visualization is an essential step in preoperative cataract assessment to rule out ocular copathologies that can adversely hidden by dense cataract such as diabetic retinopathy, age related macular degeneration or peripheral retinal tears.

B-scan ultrasonography is an important diagnostic tool used to rule out certain gross retinal pathologies such as retinal detachment, posterior staphyloma, and vitreous hemorrhage ${ }^{8}$; however, it is not adequate to check for tiny pathologies.

Preoperative fundus assessment usually done by using direct or indirect ophthalmoscopy and it may be hazy or completely indistinct in patients with dense lens opacification. Fundus examination of the fellow eye may show a hint in bilateral vitreoretinal conditions; however, some pathologies are uniocular or asymmetrical ${ }^{9}$.

In this case-series study, we developed a technique to assess posterior segment simultaneously during phacoemulsification surgery in eyes with dense cataract without modifying cataract surgery. This Endolight technique guide surgeon for an instantaneous treatment of revealed retinal co-pathologies and eliminate exposing the patient for another surgery after phacoemulsification, and theoretically expected to have a better visual prognosis for the early intervention of retinal pathology .

\section{Methods}

This case series study was carried out at Hamad Medical Corporation in Ophthalmology Department in Doha, Qatar over a period between January 2017 and June 2017.

All eyes included in the study were having dense cataract and the fundus view was hazy enough to preclude full assessment of the posterior pole, however no gross vitreoretinal pathology was indicated on B-scan ultrasonography.

All surgeries were performed by a single surgeon using the infinity phaco machine (Alcon), and phacoemulsification was performed through a temporal clear peripheral corneal incision. Anterior capsule was stained by Trypan-blue. Phacoemulsification performed in all eyes by Divide and Conquer strategy, cortical aspiration was done by bimanual probes, capsular bag was expanded by extra viscoelastic injection to accommodate endolight probe insertion (figures 1-3).

Endolight probe was inserted through either side-port into middle of capsular bag (figure 3), a vitreoretinal viewing system was used to visualize posterior segment, while posterior capsule details was visualized by endolight through microscope lens only. A single piece acrylic posterior-chamber intraocular lens was implanted in the capsular bag. Intravitreal Ranibizumab injection was required in certain cases and injected 3.5 $\mathrm{mm}$ behind superotemporal limbus.

Vitreoretinal viewing systems used: Erected Image Binocular (EIBOS) microscope, Re-sight 700 from Carl Zeiss, and Peyman-Wessels-Landers 132 D Upright Vitrectomy Lens (Figures 4-6). 


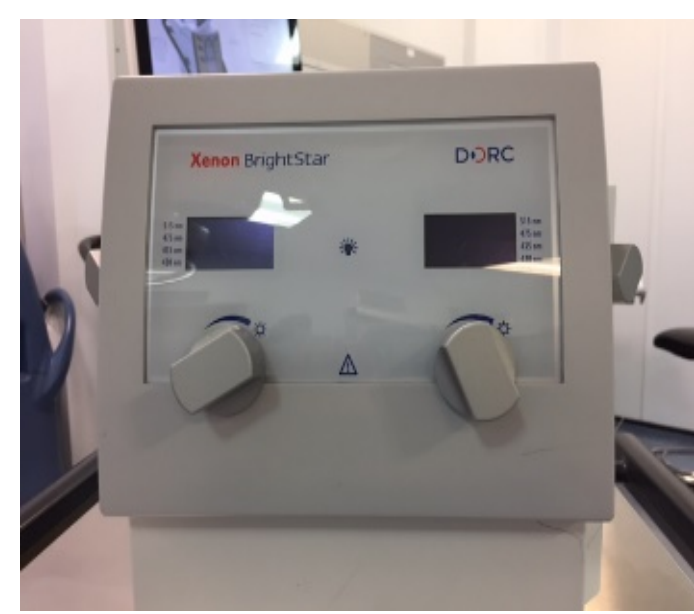

Figure 1: Xenon Bright Star Endoilluminator system 23-G from DORC

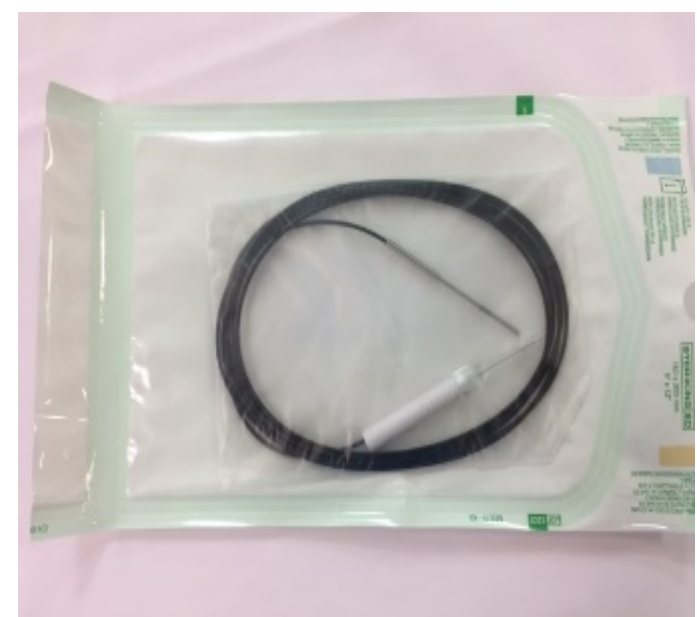

Figure 2: Endolight probe 23-G

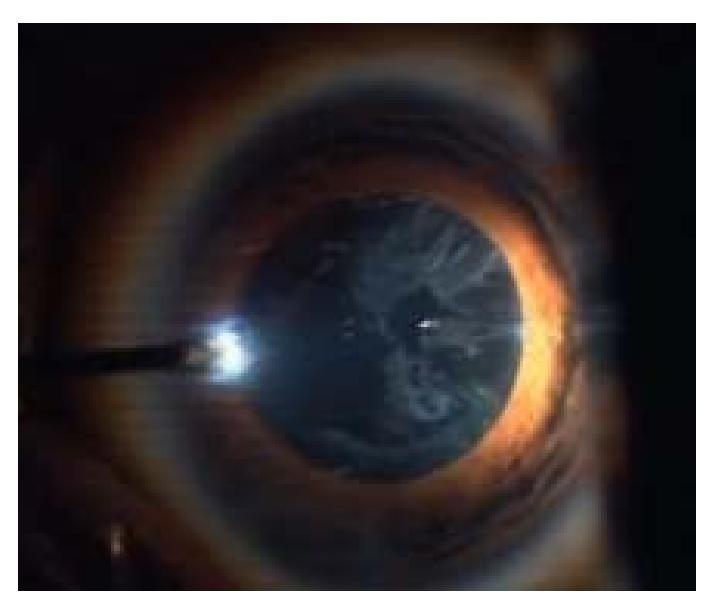

Figure 3: Less transparent posterior capsule in Translimbal Endoillumination due to direct light reflection

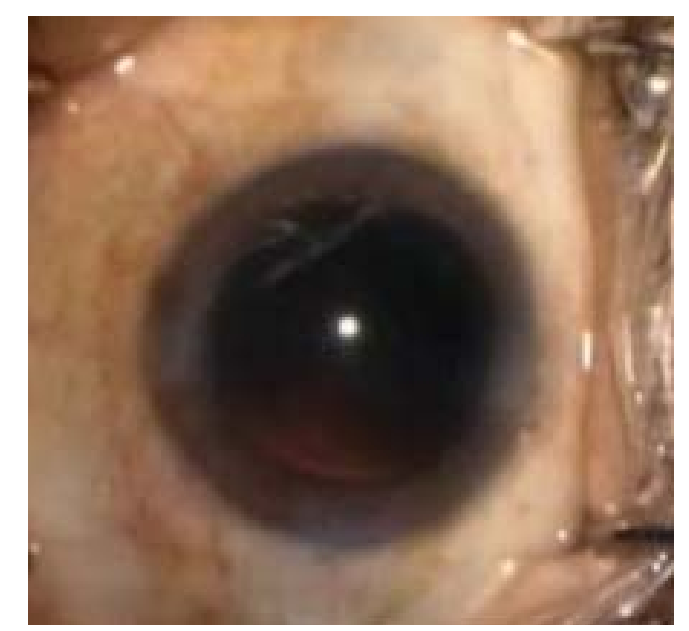

Figure 4: Posterior capsule is transparent through microscope view only
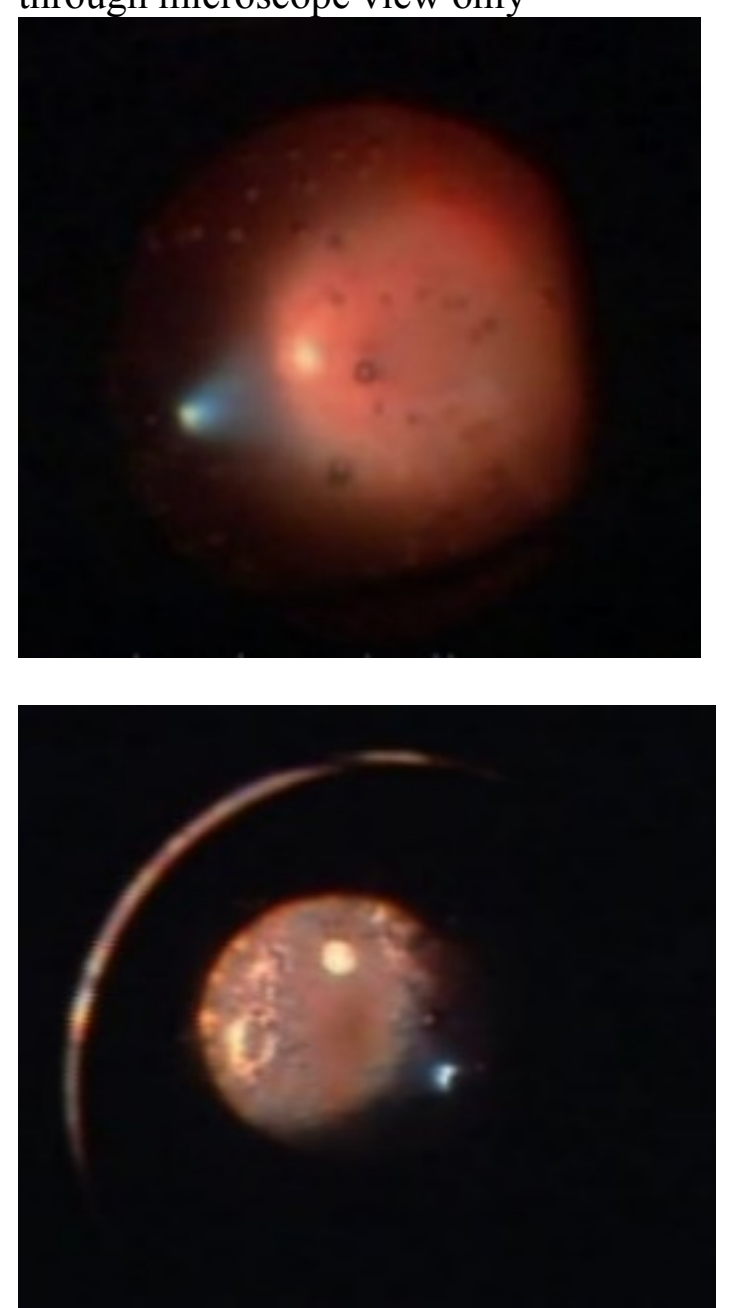

Figures 5 \& 6: Intracapsular endolight and intraoperative fundus view. 


\section{Results}

Forty-two eyes (25 Right and 17 Left) of thirty-nine patients (16 Females and 23 Males) were included in this analysis. The mean age was $64.6 \pm 13.3$ years (range: 53 to 81 years). Sixteen patients $(38 \%)$ had type-2 diabetes mellitus. No Recent history of vitreoretinal surgery recorded in all patients, however seven eyes $(17 \%)$ had intravitreal injections and all previous injections were done in a period not less than 3 months prior to cataract surgery. Topical anesthesia was applied in thirty-six eyes and subtenon anesthesia was performed in in six eyes as shown in table I. Postoperative followup of the operated eyes was done in a 30 days period.
Preoperative Visual Acuity (VA) was $\leq 6 / 60$ in all patients. Optical Coherence Tomography (OCT) imaging was not possible and obscured by dense cataract. B-Scan revealed clear vitreous and flat retina in all patients. Intraoperative Posterior Capsular thickening was detected and hence capsule polishing was done in six Eyes (14\%) (table II). Intraoperative retinal comorbidity was discovered in eight eyes (19\%); seven eyes have DME and one eye has AMD (macular drusens \& hemorrhagic spot) (table II), and all the eight eyes had simultaneous treatment by intravitreal injection of Ranibizumab during cataract surgery (table 3 ) .

Table I: Demographics and Preoperative Data

\begin{tabular}{|l|l|}
\hline Factor & Value \\
\hline Age & 53 to 81 years (Mean=64.6) \\
\hline Gender & $\begin{array}{l}\text { Male 23 }(64 \%) \\
\text { Female 16 }(36 \%)\end{array}$ \\
\hline Laterality & $\begin{array}{l}\text { Right Eye }(25)(59.5 \%) \\
\text { Left Eye }(17)(40.5 \%)\end{array}$ \\
\hline Preop. Visual Acuity $<6 / 60$ & 42 eyes (100\%) \\
\hline Diabetics & 16 patients (18 eyes) 43\% \\
\hline Previous Retinal Surgery & 0 \\
\hline Previous Intravitreal Injection & $7(16.7 \%)$ \\
\hline Anesthesia & $\begin{array}{l}\text { Topical: 36 eyes, }(85.7 \%) \\
\text { Subtenon 6 eyes, }(14.3 \%)\end{array}$ \\
\hline
\end{tabular}

Table II: Intraoperative Retina Findings

\begin{tabular}{|l|l|}
\hline Intraoperative Findings and & Number \\
\hline No retinal co-morbidity & $34(81 \%)$ \\
\hline Retinal co-morbidity & $8(19 \%)$ \\
\hline Posterior capsular thickening and then capsule polishing & $6(14 \%)$ \\
\hline
\end{tabular}

Table III: Intraoperative Interventions

\begin{tabular}{|l|l|}
\hline Retinal co-morbidity & Intraoperative Intervention \\
\hline 7 Eyes: Diabetic Macular Edema & intravitreal injection of Ranibizumab \\
\hline $\begin{array}{l}\text { 1 Eye: Hemorrhagic age } \\
\text { related macular degeneration }\end{array}$ & intravitreal injection of Ranibizumab \\
\hline
\end{tabular}




\section{Discussion}

Indirect ophthalmoscopy is the traditional method for fundus examination during cataract surgery however; the fundus view is restricted by image size (small magnification) and image inversion. We described a new technique to examine vitreoretinal compartment during phaco-surgery by translimbal insertion of endoilluminator probe and using Xenon Bright Star Endoilluminator system 23-G from DORC (Figures 1\&2). It improves view of intraocular structures during cataract surgery and has a substantial influence upon surgeon decision during this surgery to do more concomitant surgical interventions.

Fundus examination is essentials in certain patients during cataract surgery; coexisting retinal disease makes cataract surgery a greater challenge than it would normally be for the surgeon and a riskier prospect for the patient, but ophthalmologists who perform cataract surgery can minimize the impact of this extra disease burden on visual outcome by modifying their pre, peri, postoperative routines ${ }^{9}$.

It is essential to relate cataract to the degree of visual loss; the clinical examination should include a comprehensive assessment of the posterior segment. The surgeon must inform the patient that cataract surgery will only correct the cataract, but not associated undiagnosed ophthalmic conditions that could limit vision prognosis ${ }^{10}$. In this study, preoperative detailed fundus examination and macular imaging were unfeasible due to high cataract density, therefore visual acuity correlation to cataract was not possible.

In our study, all patients have been consented for cataract surgery and for possible additional surgical procedure during phaco-surgery if treatable copathology was discovered during surgery.
Several studies confirmed that Agerelated macular disease (AMD) and diabetic maculopathy were the main causes of visual disappointment after phacoemulsification ${ }^{11,12}$. By translimbal endoillumination used in this study, eight patients $(17 \%)$ revealed to have macular pathology; seven of them with diabetic macular edema and one had age-related macular degeneration, all received a prompt intravitreal Ranibizumab injection during phacoemulsification.

All surgeries in this study were done by the same surgeon, under topical anesthesia except for six cases by subtenon anesthesia.

Two main steps of technique: Intracameral (Inside Anterior Chamber) endolight insertion with use of normal microscope lens viewing system: this step improved visualization of posterior capsule (PC) as it looks less transparent (Figure 3), however PC is usually look transparent by direct microscope illumination only without endoilluminator (Figure 4). Theoretically, it is due to direct endo light reflection from anterior surface of PC (Figure 3). This technique helps to confirm if PC integrity was questionable.

Intracapsular endolight insertion after capsular bag expansion by viscoelastic prior to IOL implantation and use of vitreoretinal viewing system (Figures $5,6)$.

Fundus view in translimbal endoillumination is comparable to trans-scleral endoillumination during pars plana vitrectomy except for a limited manipulation of the probe within the capsular bag.

The type of the vitreoretinal viewing system used could affect the quality of fundus view obtained by translimbal endoillumination, in this study 3 kinds of viewing systems were used; EIBOS Erected Image Binocular microscope, Re- 
sight 700 from Carl Zeiss, PeymanWessels-Landers, 132 D Upright Vitrectomy Lens. The fundus view was superior in Zeiss resight viewing system. Re-sight 700 from Carl Zeiss is easier, gives good working space between the viewing lens and the endoprobe, and has wider and clearer fundus view.

The OCT is a new technology to assist macular scanning in vitreoretinal surgeries, however it is rather expensive and it requires the surgeon to halt surgery prior to performing the scan and results in increased surgical time ${ }^{13}$.
In conclusion, translimbal endoillumination during phacoemulsification has an improved view to fundus and posterior capsule. Image size and resolution were controlled by vitreoretinal viewing system used and microscope. No extra incision was required for endoillumination and it is cost effective. Translimbal endo-illumination technique guides the surgeon to have a prompt decision for more surgical intervention if retinal co-pathology was discovered. This technique add a refining step to phacosurgery by allowing a detailed assessment of the posterior capsule.

\section{References}

1. Thomas Kohnen, Martin Baumeister, Daniel Kook, Oliver K. Klaproth, Christian Ohrloff. Cataract Surgery With Implantation of an Artificial Lens. Dtsch Arztebl Int. 2009 Oct; 106(43): 695-702

2. Jaycock P, Johnston RL, Taylor H. The Cataract National Dataset electronic multi-centre audit of 55,567 operations: updating benchmark standards of care in the United Kingdom and internationally. Eye (Lond). 2009;23(1):38-49.

3. Hong T1, Mitchell P, de Loryn T, Rochtchina E, Cugati S, Wang JJ. Development and progression of diabetic retinopathy 12 months after phacoemulsification cataract surgery.Ophthalmology 2009;116(8):1510-1514

4. Freeman EE1, Munoz B, West SK, Tielsch JM, Schein OD. Is there an association between cataract surgery and age-related macular degeneration? Data from three population-based studies.. Am J Ophthalmol 2003;135(6):849-856

5. Li Ming Dong, Walter J. Stark, Joan L. Jefferys. Progression of Age-Related Macular Degeneration After Cataract Surgery. Arch Ophthalmol 2009;127(11):1412-1419

6. Ronald Klein, MD, MPH; Barbara E. K. Klein, MD; Tien Y. Wong, MD; et al.The Association of Cataract and Cataract Surgery With the Long-term Incidence of Age-Related Maculopathy Arch Ophthalmol 2002;120(11):1551-1558.

7. Haynie M, Retinal Considerations Prior to Cataract Surgery, Preview of Optometry, October 1,2015

8. Anteby II, Blumenthal EZ, Zamir E, Waindim P. The role of preopera-tive ultrasonography for patients with dense cataract: a retrospective study of 509 cases. Ophthalmic Surg Lasers. 1998;29(2):114-118.

9. Linda Roach, Kimberly A. Drenser, Thomas A. Oetting, Adrienne Williams Scott. Special Considerations in Cataract Surgery: Coexisting Retinal Disease. Clinical Update. EYENET 27

10. Kalpna B. N. \& Murali B. Cataract and posterior segment risk factors. Med. Res. Chron., 2015, 2 (2), 226-234

11. Chatziralli IP, Kanonidou E, Papazisis L. Frequency of fundus pathology related to patients' dissatisfaction after phacoemulsification cataract surgery. Bull Soc Belge Ophtalmol. 2011;317:21-24

12. Monestam E, Wachtmeister L. Dissatisfaction with cataract surgery in relation to visual results in a population-based study in Sweden. J Cataract Refract Surg. 1999;25(8):1127-1134. 13. Justis P. Ehlers, Yuankai K. Tao, Sunil K. Srivastava. The Value of Intraoperative OCT Imaging in Vitreoretinal Surgery. Curr Opin Ophthalmol. 2014 May ; 25(3): 221-227. 